\title{
A valveless micro impedance pump driven by electromagnetic actuation
}

\author{
Derek Rinderknecht ${ }^{1}$, Anna Iwaniec Hickerson ${ }^{1}$ \\ and Morteza Gharib ${ }^{2}$ \\ ${ }^{1}$ Option of Bioengineering, California Institute of Technology, MC 205-45, \\ 1200 E. California Blvd., Pasadena, CA 91125, USA \\ ${ }^{2}$ Hans W Liepmann Professor of Aeronautics and Bioengineering, California Institute of \\ Technology, MC 205-45, 1200 E. California Blvd, Pasadena, CA 91125, USA \\ E-mail: rinderkn@caltech.edu
}

Received 25 October 2004, in final form 21 January 2005

Published 11 March 2005

Online at stacks.iop.org/JMM/15/861

\begin{abstract}
Over the past two decades, a variety of micropumps have been explored for various applications in microfluidics such as control of pico- and nanoliter flows for drug delivery as well as chemical mixing and analysis. We present the fabrication and preliminary experimental studies of flow performance on the micro impedance pump, a previously unexplored method of pumping fluid on the microscale. The micro impedance pump was constructed of a simple thin-walled tube coupled at either end to glass capillary tubing and actuated electromagnetically. Through the cumulative effects of wave propagation and reflection originating from an excitation located asymmetrically along the length of the elastic tube, a pressure head can be established to drive flow. Flow rates were observed to be reversible and highly dependent on the profile of the excitation. Micro impedance pump flow studies were conducted in open and closed circuit flow configurations.

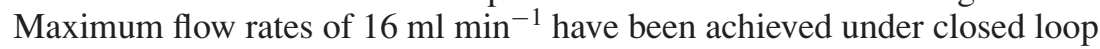
flow conditions with an elastic tube diameter of $2 \mathrm{~mm}$. Two size scales with channel diameters of $2 \mathrm{~mm}$ and $250 \mu \mathrm{m}$ were also examined in open circuit flow, resulting in flow rates of $191 \mu \mathrm{l} \mathrm{min}^{-1}$ and $17 \mu 1 \mathrm{~min}^{-1}$, respectively.
\end{abstract}

\section{Introduction}

Recent interest in microfluidics and microfluidic devices has been predominately driven by the need for biomedical devices on the microscale as well as applications involving chemical control, mixing and analysis, which stem from the push toward lab-on-chip (LOC) methodologies. Micropumping is a necessary component of large integrated systems for sample control and mixing. A micropump requires a compact method of actuation and a mechanism to produce the flow. Commonly micropumps are driven by piezoelectric, electrostatic, electromagnetic, electrohydrodynamic or pneumatic actuators. Mechanisms of pumping vary greatly but can generally be grouped into two categories: displacement pumps and dynamic pumps [1]. These mechanisms can further be categorized in a variety of ways; one of which is the presence of valves. Conventional valves in microfluidics systems are subject to mechanical failure and, in the case of biological fluids, present further risk of malfunction due to clogging or to damage sensitive biomolecules. Current valveless pumping techniques mainly consist of peristaltic [2-5] and reciprocating diaphragm pumps relying on diffusers [6-10]. These systems are often fabricated on a substrate through the use of soft lithography on polymeric materials because they are flexible and allow the form and features of these devices to be created and remain functionally sound. Substrate-based systems, however, occupy much more volume than is actually required by the device.

Here, we present a new valveless and substrate-free impedance-based technique for pumping fluid on the microscale. It should be noted that the phenomena resulting in impedance-defined flows has been known for quite some time [11-15]. However, this study is the first of its kind to demonstrate the feasibility of pumping with these phenomena on the microscale, under two different flow circuit configurations, and on two different size scales, 
Table 1. A summary of the impedance pump testing configurations including actuation type and dimensions based on the testing configuration.

\begin{tabular}{llll}
\hline Configuration & $\begin{array}{l}\text { Tubing dimensions } \\
\text { ID/wall }\end{array}$ & Material & Actuation type \\
\hline Closed loop & $2 \mathrm{~mm} / 50 \mu \mathrm{m}$ & Silicone & Perpendicular (figure 3) \\
Open loop & $2 \mathrm{~mm} / 50 \mu \mathrm{m}$ & Silicone & In-line (figure 4) \\
Micro open loop & $250 \mu \mathrm{m} / 50 \mu \mathrm{m}$ & Polyurethane & Perpendicular (figure 3) \\
\hline
\end{tabular}

Impedance Pump Schematic

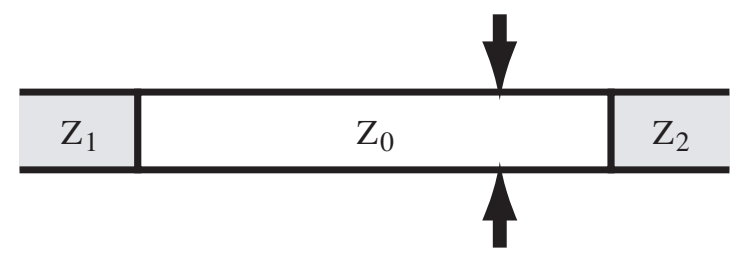

Figure 1. A schematic of an impedance pump. The impedances are represented by $Z_{0}, Z_{1}$ and $Z_{2}$ respectively. The arrows designate the location of the excitation.

as well as report quantitative measurements of the flow performance.

\section{Principle of operation}

An impedance pump is comprised of a compressible section, usually a tube, coupled at both ends to end members of different impedances. Since impedance-based pumping is dependent on wave reflection, any material allowing for wave propagation is suitable for the compressible section; in experiments, this section is commonly made of a silicone or latex tube. Coupling this flexible tubing at either end to another material or materials of different mechanical properties, geometries or any other factor affecting wave propagation and/or reflection, creates an impedance mismatch, and therefore a site for wave reflection. In figure 1, which depicts a schematic of the pump, these impedances are represented by $\mathrm{Z}_{0}, \mathrm{Z}_{1}$ and $\mathrm{Z}_{2}$. The most fundamental principle of its operation is that the excitation occurs asymmetrically with respect to the impedance of the fluid system. Periodic pinching at an asymmetric location at a certain frequency, waveform and duty cycle results in the accumulation of a pressure gradient from wave interference and therefore the potential to drive flow.

In our experiments, this system takes the form of a thinwalled elastic tube coupled at either end to two symmetric segments composed of a less compliant material, glass and filled with water. Since the glass segments are of equal impedance, the asymmetry must be caused by the location of the excitation along the length of the elastic tube. The difference in length over which the waves travel before reflecting imposes a phase difference in the travelling waves returning to the excitation site. Neglecting attenuation and the effects of viscosity for simplicity, one could imagine conditions where at a certain frequency or band of frequencies the interference of reflecting waves travelling in both directions can form pressure gradients and induce flow. Through the complete collapse of the elastic section the system can also function as a valve.

\section{Experimental set-up}

For the remainder of this paper, the following distinction will be made. Open loop will be defined as flow between two independent reservoirs on both sides of the pump, with no fluidic interconnections. Closed loop will be defined as flow within a distinct loop. Previously, the behavior of the impedance pump has been studied in both of these configurations at larger size scales [16]. In this study, the impedance pump was tested under three configurations spanning two different size scales: closed loop, open loop and micro open loop. For these configurations, two different electromagnetic actuation schemes were devised referred to as perpendicular and in-line, specifying their orientation with respect to the axis of the fluid channel. Table 1 summarizes the configurations and their respective electromagnetic actuator types. In all cases, the glass sections attached to the micro pump are symmetric, and therefore equal in impedance. As a result of this symmetry, excitation at the midline of the elastic tube produces no net flow.

The thin-walled elastic tubes used for the pump in the open loop and closed loop experiments were made of silicone with a Young's modulus of $0.4 \mathrm{MPa}$ (at $100 \%$ elongation), an inner diameter (ID) of $2 \mathrm{~mm}$ and a wall thickness of $50 \mu \mathrm{m}$. The tubing used in the micro open loop study was made of polyurethane with a Young's modulus of $2 \mathrm{MPa}$ (at $100 \%$ elongation), an inner diameter of $250 \mu \mathrm{m}$ and a wall thickness of $50 \mu \mathrm{m}$. Unless otherwise mentioned, the elastic tubing was connected to glass tubing with an inner diameter of $0.8 \mathrm{~mm}$ and a $0.6 \mathrm{~mm}$ wall thickness. All impedance pumps constructed using the tubing mentioned in the previous sentence were sealed on either end to the glass tube using heat shrink. A diagram of the assembly procedure can be seen in figure 2 .

\subsection{Micro impedance pump actuation}

Although any technique could have been employed, electromagnetic actuation was chosen because of its large displacement, high-frequency capabilities. Two different electromagnetic actuation schemes were examined, one for experimental purposes and the other for device-oriented studies, as shown in figures 3 and 4, respectively. Actuation was achieved by fixing a neodymium iron boron $(\mathrm{NdFeB})$ rare earth magnet cube with a side length of $2.4 \mathrm{~mm}$ using silicone glue to the upper surface of the tube at an asymmetrical location along the elastic tube length. This magnet was oriented such that when the coil was energized their magnetic field gradients produce a radial force compressing the tube. In the first configuration, the magnetic field of the magnet was oriented such that when the coil was energized its poles are parallel to those of the permanent magnet. A schematic illustrating the 


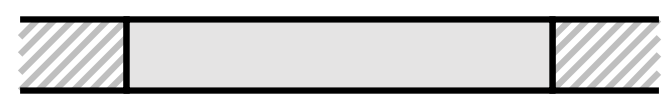

2

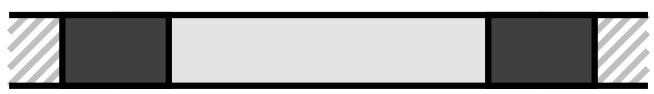

3

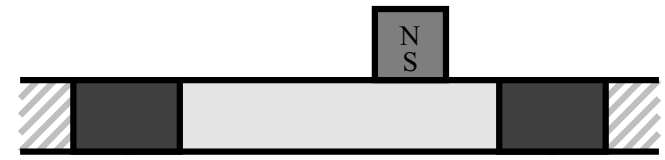

4

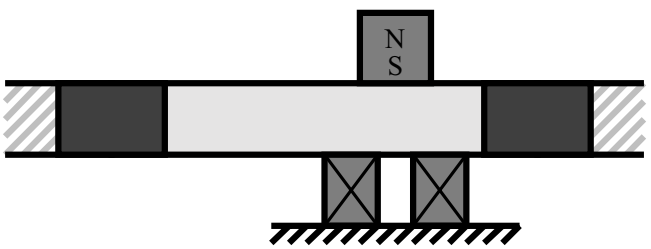

A thin-walled elastic tube is coupled

at both ends to glass capillary tubing.

Heat shrink is used to seal the elastic tube onto the capillary tubes.

Silicone sealant is applied to fix a

$\mathrm{NdFeB}$ magnet to the elastic section.

An inductive coil is centered under the magnet.

Figure 2. A diagram showing the assembly of an electromagnetically actuated impedance pump.

Perpendicular Actuator

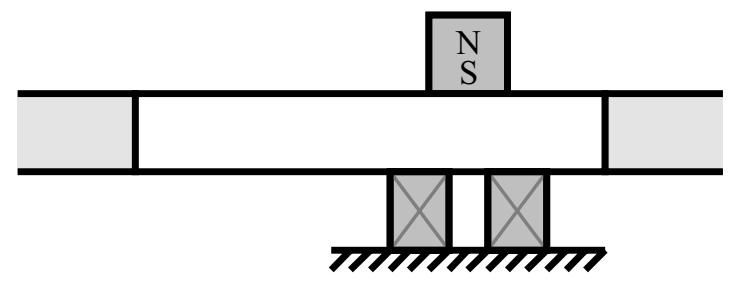

Figure 3. Schematic showing an impedance pump with inductance-driven actuation in the perpendicular configuration. The letters $\mathrm{N}$ and $\mathrm{S}$ designate the orientation of the permanent magnet poles.

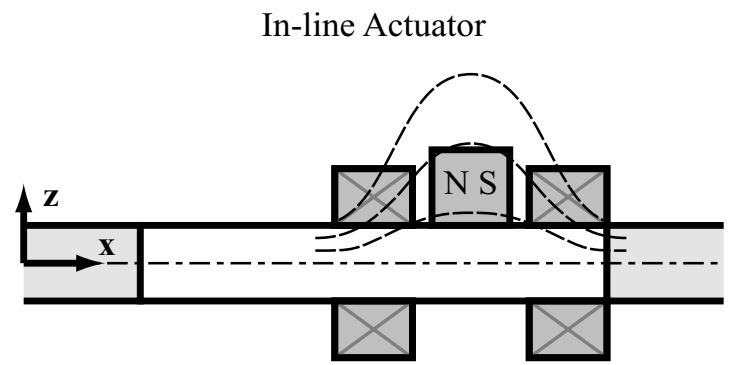

Figure 4. A schematic of the inductive coils wound in the in-line azimuthally symmetric format parallel to the $x$-axis along which flow occurs. The letters $\mathrm{N}$ and $\mathrm{S}$ designate the orientation of the permanent magnet poles.

inductor-driven electromagnetic actuator in a perpendicular configuration can be seen in figure 3 .

The second actuation scheme takes advantage of the fact that fluid passages in biological systems as well as medical devices are generally cylindrically symmetric in shape. To conserve space as well as complement the symmetry of the tube, a novel inline coil-magnet configuration was created comprised of two coils wound azimuthally symmetric with respect to the elastic tube and connected such that when energized they are in-phase. A compressive radial force can therefore be generated by locating a permanent magnet element equidistant from both coils with its magnetic pole oriented such that it is parallel to the length of the tube. A diagram of the inline coil-magnet configuration can be seen in figure 4 .

\subsection{Closed loop flow}

The closed loop test section was formed by coupling a thinwalled elastic tube to one end of the two symmetric sections of the glass flow loop. The fluidic loop was completed by connecting the opposite ends to a latex tube with a significantly thicker wall, making it much more rigid than the elastic tube of the pump but soft enough to be crimped thereby increasing the fluid resistance in the loop. Crimping this thicker elastic tube completely closes one section off from the other and creates an open loop situation in which a pressure differential can be measured between the water levels on either side of the pump. Each section also has a piece of glass tubing connected perpendicular to the plane of the flow loop on the opposite end of the pumping site. The inner diameter along a majority of the length of the glass flow loop is $3.2 \mathrm{~mm}$. On one end, the tubing of the loop tapers to accommodate a $2 \mathrm{~mm}$ side-length square section, where the flow rates can be measured. The square section is then connected to a smaller diameter glass tube $(0.8 \mathrm{~mm} \mathrm{ID}$, $0.6 \mathrm{~mm}$ wall) that connects the loop to the elastic tube. Figure 5 shows a picture of the closed loop flow circuit. In this configuration, energizing an inductive coil positioned under the magnet allows the tube to be compressed forming the pump. A schematic of the actuator can be seen in figure 3.

Measurements of the bulk flow velocity were taken using a Viosense mini laser Doppler velocimetry (LDV) system through the length of square tubing on the flow loop. The fluid was seeded with $3 \mu \mathrm{m}$ neutrally buoyant polystyrene microspheres. The best results were achieved with particles equal to or less than the fringe spacing $(\sim 11 \mu \mathrm{m})$ of the LDV. Important parameters for reliable measurements and to prevent 


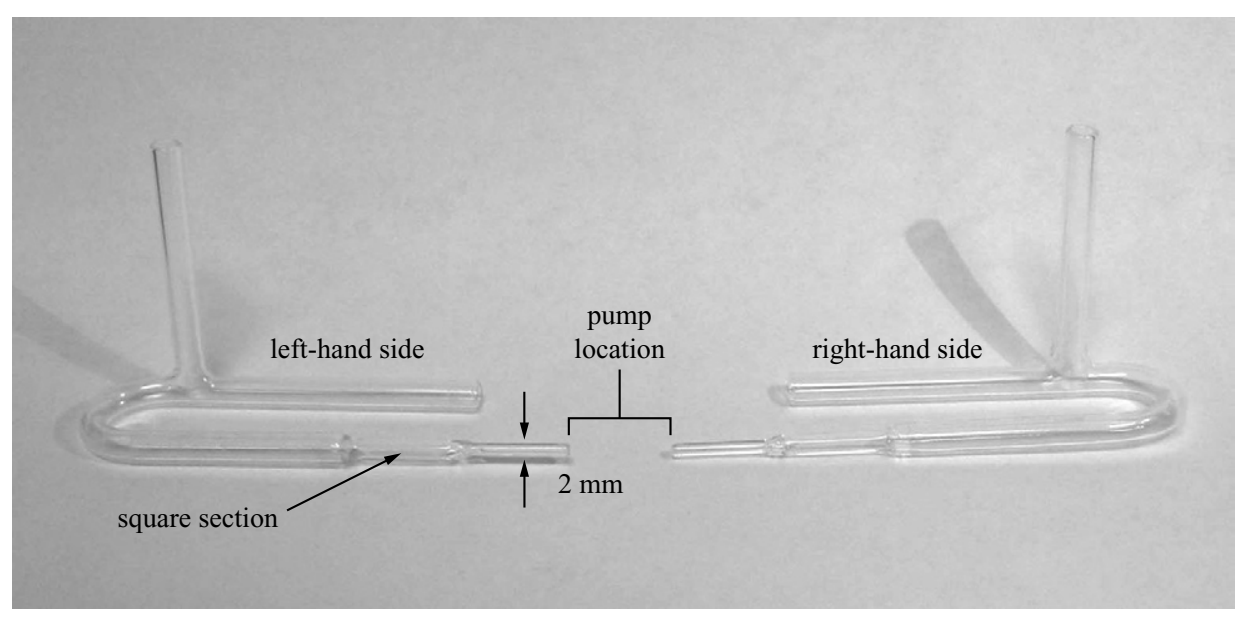

Figure 5. A picture of the closed loop flow circuit glass pieces. In operation the pump is attached between the two sides of the loop closest to the reader with an outer diameter of $2 \mathrm{~mm}$. Also shown is the square section where flow velocity is measured. For measurements, clockwise flow within the loop, from the right-hand side to the left-hand side, was taken as positive.

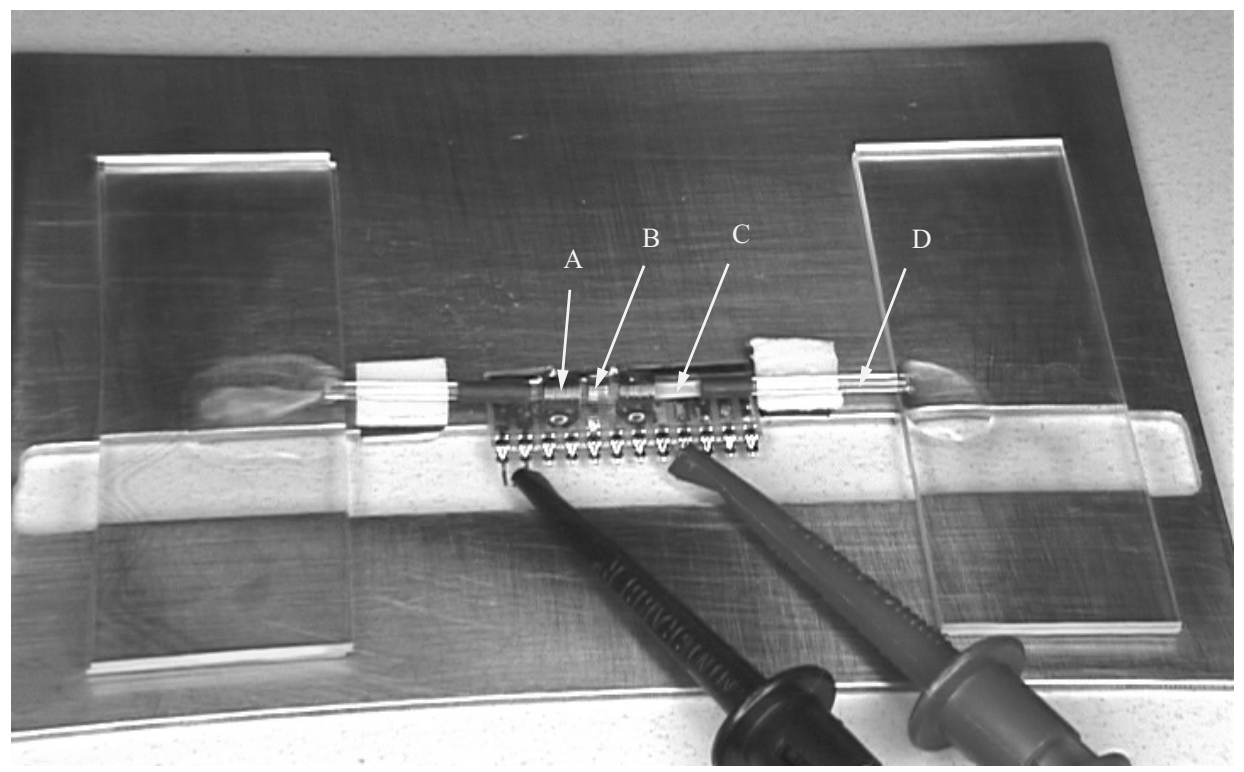

Figure 6. The functioning pump in the open loop configuration employing the in-line actuation technique. The letters A, B, C and D denote the coil, magnet, silicone tube and glass tube, respectively. Fluorescein dye has been used to visualize the movement of fluid from one reservoir to the other. The Teflon slides have been removed for clarity.

the particles from adhering to the glass flow loop were the particle size, material and buoyancy.

\subsection{Open loop flow}

In a device-driven study similar to the environment under which a medically implantable device may operate, the inflow to the device may not contain returning fluid inertia. Open loop flow was therefore demonstrated by connecting the silicone tubing to a short length of the glass tubing $(0.8 \mathrm{~mm} \mathrm{ID \text {, }}$ $0.6 \mathrm{~mm}$ wall) through the procedure described in figure 2 and placing either end into two independent water reservoirs of a known volume, dispensed through a micropipette. Flow rates were determined by measuring the time required for the pump to consume the entire droplet. To prevent capillary action from drawing the fluid along the bottom of the tube, the ends of the glass tubing were placed on two glass slides disconnected from the pump as well as each other. In addition, polytetrafluoroethylene (PTFE) rectangles were stuck to the slides using double-sided tape. The hydrophobicity of PTFE facilitates the visualization of the droplets. An image of such an assembly can be seen in figure 6 .

\subsection{Micro open loop}

The micro open loop flow experiment was constructed by pressing a short length of glass capillary tubing into either end of the elastic section. In this case, heat shrink was not required to seal the coupled tubes, as described by the procedure above. Similar to the larger open loop study Teflon-coated slides were used to visualize the flow of droplets of known volume from one side of the pump to the other. The excitation in this case was provided by cantilever style actuator based on the in-line electromagnetic scheme. A picture of the micro open loop test assembly can be seen in figure 7 . 


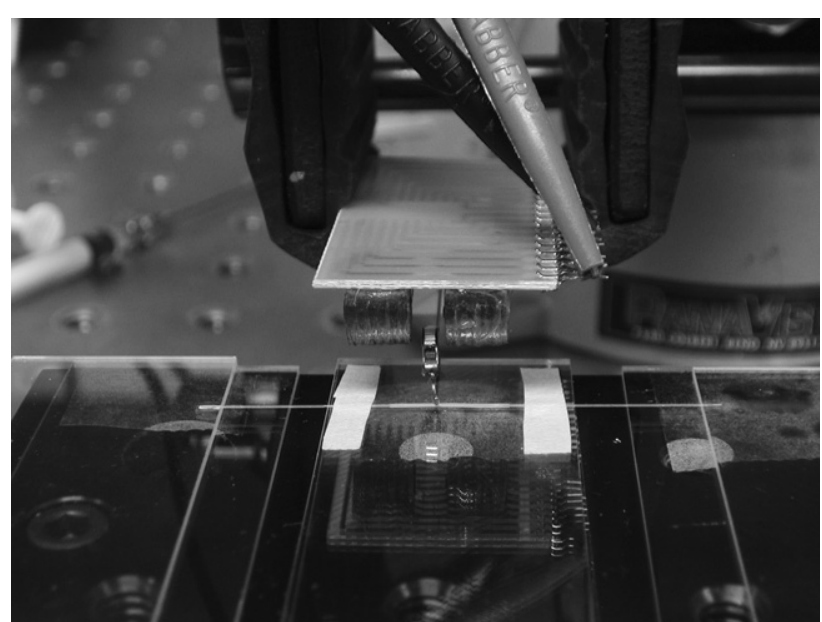

Figure 7. Picture of the micro open loop configuration showing the elastic section, glass tubing and actuator. The PTFE slides are not present so the glass tubing is visible.

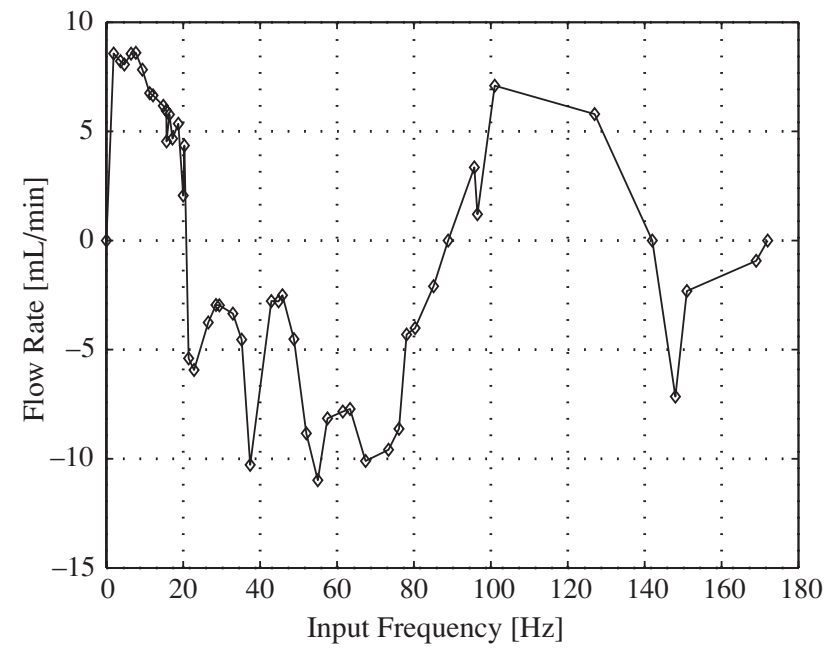

Figure 8. A typical frequency flow response of an impedance pump, comprised of an elastic section with an inner diameter of $2 \mathrm{~mm}$ and wall thickness of $50 \mu \mathrm{m}$ coupled at either end to glass tubing of $0.8 \mathrm{~mm}$ in inner diameter and $0.6 \mathrm{~mm}$ wall thickness excited $4.2 \mathrm{~mm}$ from the right-hand side of the flow loop.

\section{Results}

\subsection{Closed loop}

The closed loop flow response of the impedance pump was observed to be reversible as well as highly dependent on frequency. The performance of the pump was found to be very sensitive to the waveform, offset, amplitude and duty cycle of the excitation. A typical frequency response can be seen in figure 8 . The data points in the plot in figure 8 represent an average of 100 samples taken at the midline of the flow. Clockwise flow, into the right-hand side of the elastic tube, was taken as positive. The magnet was located at $4.2 \mathrm{~mm}$ from the right-hand side of the $19.6 \mathrm{~mm}$ long elastic tube. The input to the coil was a square waveform, $48 \mathrm{~mA}$ in amplitude, with a $16 \mathrm{~mA}$ offset and a $50 \%$ duty cycle. Consistently, there was at least one flow reversal as the frequency was increased. Figure 8 shows three such reversals. These can be seen to occur at roughly $21 \mathrm{~Hz}, 89 \mathrm{~Hz}$ and $142 \mathrm{~Hz}$. Although similar net flow rates were observed at both high and low frequencies, at low frequencies the flow was highly oscillatory. As the frequency of excitation was increased, bulk flow oscillations tend to damp out resulting in unidirectional flow. Flow rates of up to $16 \mathrm{ml} \mathrm{min}^{-1}$ have been achieved. For the curve shown in figure 8 , the maximum flow rate was $11 \mathrm{ml} \mathrm{min}^{-1}$ at $55 \mathrm{~Hz}$ corresponding to a Reynolds number $(R e)$ of 730 .

\subsection{Open loop}

In the open loop configuration, the magnet was located $11.9 \mathrm{~mm}$ from the right-hand side of the $19.1 \mathrm{~mm}$ long elastic tube. The input to the coils was a $60.7 \mathrm{~Hz}$, a square wave $200 \mathrm{~mA}$ in amplitude with no offset. Flow rates exceeding $191 \mu 1 \mathrm{~min}^{-1}$ were achieved pumping a $100 \mu \mathrm{l}$ droplet to the opposite end of the pump, corresponding to a $R e$ of 5 . Relatively, high flow was achieved despite the fact that, in this set-up, the magnet does not in any form compress the tube against a fixed opposing surface.

\subsection{Open loop micro}

Since viscosity tends to dominate on the microscale, a study was done to determine whether impedance defined flows still function at very low Re. Tests were performed in the open loop configuration described above using a polyurethane (PU) tube with a $250 \mu \mathrm{m}$ inner diameter and a $50 \mu \mathrm{m}$ wall thickness coupled to a borosilicate glass capillary tube with a $150 \mu \mathrm{m}$ inner diameter and a $50 \mu \mathrm{m}$ wall thickness. PU was chosen due to its large tensile strength when compared to most elastomers enabling smaller inner diameters and wall thicknesses than would otherwise be possible. Using an $82.0 \mathrm{~Hz}$ excitation and water as the fluid, flow rates were measured to be approximately $17 \mu \mathrm{l} \mathrm{min}^{-1}$ or a $R e \sim 2$. The pressure gradient created by the pump was large enough to expel droplets out of one end of the test assembly. In this configuration, flow has been shown to be highly frequency dependent and reversible.

\section{Discussion}

High-frequency excitation revealed the presence of multiple regimes of flow. The upper limit of this excitation was only limited by the attenuation of the magnetic field gradients produced by the coil at high frequency, consequently changing the excitation profile. Flow in all cases was observed to be highly dependent on frequency, waveform, duty cycle and amplitude of the input excitation. The reliance of impedance-driven flows on the type of excitation provides some evidence for the occurrence of wave-based interactions being cumulatively responsible for the manifestation of flow. The dependence of the flow rate on frequency can be observed in figure 8. Wave interference also offers some explanation as to why there was consistently a flow reversal. The presence of the elastic section implied that there was at least some phase delay between the pressure and velocity waves produced with each excitation. The flow reversals as well as the flow peaks most likely correspond to frequencies where the phase delay between the reflecting waves and the source 
waves was the least and most optimal, respectively. The vast frequency range for which flow occurs allows for the selection of completely different, application-specific flow regimes with a single pump.

Functionality in the open loop configuration is also significant. In essence, the inertia of the returning flow is not required to maintain pressure or flow. Instead, solely the presence of an impedance mismatch and asymmetric excitation are required to generate a pressure gradient at certain frequencies. The flow rates in either case differ by two orders of magnitude. As reported above, the micro impedance pump in the closed loop flow configuration has

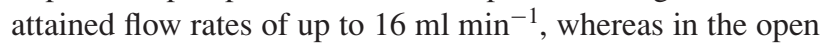
loop configuration under similar parameters maximum flow rates have consistently been much lower $\left(\sim 191 \mu 1 \mathrm{~min}^{-1}\right)$. In contrast to other mechanisms of pumping, the increased system impedance in the closed loop configuration may actually benefit the impedance pump's performance.

\section{Conclusion}

Impedance-based pumping has been shown to be a viable method for moving and controlling fluids on the microscale. High-frequency electromagnetic actuation has revealed multiple regimes of performance which can be controlled as well as reversed by simply varying the frequency of excitation. Flow rates of $16 \mathrm{ml} \mathrm{min}{ }^{-1}$ and $191 \mu 1 \mathrm{~min}^{-1}$ have been demonstrated in the closed loop and open loop flow configurations respectively. In the micro open loop trials, flow rates as high as $17 \mu 1 \mathrm{~min}^{-1}$ have been achieved.

The performance of the micro impedance pump has been observed to be dependent on a variety of parameters, namely the duty cycle, frequency and amplitude of excitation as well as the fluid-mechanical properties of the system. Adjusting one parameter, such as the frequency, over a very small range enables the device to produce a broad range of output flows. The micro impedance pump is relatively easy to construct and does not require any complex fabrication methods to build and operate. We expect that the micro impedance pump will prove to be highly resistant to clogging due in part to the pulsatile nature of the flow as well as the lack of diffusers and valves. The presence of flow in the absence of complete closure of tube, in both the open loop and closed loop configurations, may prove to be beneficial for the handling of sensitive biofluids. In addition to its substrate-free construction, the absence of complex parts and inner geometries makes the impedance pump well suited for integration into cost-focused, spacelimited applications including drug delivery, cell sorting, chemical analysis as well as act to complement current stenting and shunting techniques. Micro impedance-based pumping presents an exciting new method of controlling fluids on the microscale. Although it is conceptually simple, significant future work is necessary to be able to further understand and predict the complex dynamics of impedance-driven flows.

\section{Acknowledgments}

We would like to give special thanks to the Gordon and Betty Moore Fellowship, Benjamin J. Rosen Fellowship, the Grubstake Fund and the Fehrer Fellowship for providing support for this project. Additionally, we would like to thank Professor Mladen Barbic for his advice on electromagnetic actuation as well as Apex Microtechnology for their generous contribution of electronic components.

\section{References}

[1] Laser D J and Santiago J G 2004 A review of micropumps J. Micromech. Microeng. 14 R35-64

[2] Unger M A, Chou H P, Thorsen T, Scherer A and Quake S R 2000 Monolithic microfabricated valves and pumps by multilayer soft lithography Science 288 113-6

[3] Quake S and Scherer A 2000 From micro- to nanofabrication with soft materials Science 290 1536-40

[4] Berg J M, Anderson R, Anaya M, Lahlouh B, Holtz M and Dallas T 2003 A two-stage discrete peristaltic micropump Sensors Actuators A 104 6-10

[5] Husband B, Bu M, Evans A G R and Melvin T 2004 Investigation for the operation of an integrated peristaltic micropump J. Micromech. Microeng. 14 S64-9

[6] Olsson A, Stemme G and Stemme E 1995 A valve-less planar fluid pump with two pump chambers Sensors Actuators A 46-47 549-56

[7] Gerlach T and Wurmus H 1995 Working principle and performance of the dynamic micropump Sensors Actuators A 50 135-40

[8] Olsson A, Enoksson P, Stemme G and Stemme E 1996 A valve-less planar pump isotropically etched in silicon J. Micromech. Microeng. 6 87-91

[9] Andersson H, van der Wijngaart W, Nilsson P, Enoksson P and Stemme G 2001 A valve-less diffuser micropump for microfluidics analytical systems Sensors Actuators B 72 259-65

[10] Schabmueller C G J, Kock M, Mokhtari M E, Evans A G R, Brunnschweiler A and Sehr H 2002 Self-aligning gas/liquid micropump J. Micromech. Microeng. 12 420-4

[11] Liebau G 1954 Über ein ventilloses Pumprinzip Naturwissenschaften $\mathbf{4 1} 327$

[12] Liebau G 1955 Die Strömungsprinzipien des Herzens Zs. Kreislaufforshung 44 677-84

[13] Thomann H 1978 A Simple pumping mechanism in a valveless tube J. Appl. Math. Phys. 29 169-77

[14] Moser M et al 1998 Impedance defined flow generalization of William Harvey's concept of circulation-370 yrs later Int. J. Cardiovasc. Med. Sci. 1 205-11

[15] Auerbach D, Moehring W and Moser M 2004 An analytic approach to the Liebau problem of valveless pumping Cardiovasc. Eng. Int. J. 4 201-7

[16] Hickerson A I, Rinderknecht D and Gharib M 2005 Experimental study of a valveless impedance pump Exp. Fluids at press 